\title{
Zika Pregnancy Surveillance: Transforming Data into Educational and Clinical Tools
}

\section{Kara Polen, Titilope Oduyebo, Jazmyn Moore, Sascha Ellinton, Regina Simeone, Samantha Olson, Margaret A. Honein, Dana Meaney-Delman}

Centers for Disease Control and Prevention, United States

Objective

To describe how Zika virus (Zika) surveillance data informs and improves testing guidance, clinical evaluation and management of pregnant women and infants with possible Zika infection

Introduction

Little was known about the maternal and fetal/infant effects of Zika infection before the 2015 outbreak in the Americas, which made it challenging for public health practitioners and clinicians to care for pregnant women and infants exposed to Zika. In 2016, CDC implemented a rapid surveillance system, the US Zika Pregnancy and Infant Registry, to collect information about the impact of Zika infection during pregnancy and inform the CDC response and clinical guidance. In partnership with state, tribal, local, and territorial health departments, CDC disseminated information from this surveillance system, which served as the foundation for educational materials and clinical tools for healthcare providers.

\section{Methods}

Throughout the Zika response, CDC worked closely with health officers, epidemiologists, and clinical partners to seek expert input on the interpretation of emerging data and the evaluation and management of these vulnerable populations. In response to requests from clinical and public health partners, CDC created targeted educational materials and tools to facilitate the implementation of clinical guidance. These materials equipped healthcare providers with the information needed to care for pregnant women and infants with Zika infection. Examples of products developed included: 1) screening tools to identify pregnant women for whom testing is indicated; 2) an interactive web tool to assist with implementation and interpretation of Zika testing guidance (Pregnancy and Zika Testing Widget); 3) patient counseling scripts; and 4) videos to explain critical clinical concepts (e.g., measurement of infant head circumference). These tools were informally pre-tested with the target audiences prior to dissemination, specifically to assess usefulness in clinical settings. CDC disseminated these tools through the CDC website and through comprehensive outreach (e.g., webinars, calls, email alerts) to various audiences. Additionally, several professional organizations incorporated these tools into regular communication with their membership.

\section{Results}

The US Zika Pregnancy and Infant Registry is currently monitoring infants from approximately 7,300 pregnancies in the US states and territories with laboratory evidence of Zika. Surveillance data provided valuable information, including clues toward the pattern of defects and other neurologic disabilities associated with congenital Zika infection, estimates of the risks associated with congenital infection, and timeframes of greatest risk during pregnancy, to help clinicians counsel pregnant patients with potential Zika exposure. CDC used these data to inform their clinical tools, particularly in pretest counseling materials and educational factsheets for healthcare providers to use with pregnant women with potential Zika exposure.

After informal testing among healthcare providers, the tools received positive feedback regarding usefulness and applicability in clinical settings. Collectively, CDC's Zika clinical tools were downloaded more than 300,000 times from CDC's website. The Pregnancy and Zika Testing Widget was accessed and followed to an endpoint (e.g., Zika testing recommended) more than 17,000 times, with more than $75 \%$ of users self-identifying as clinicians.

\section{Conclusions}

Rapid implementation of Zika surveillance captured evolving data about the impact of Zika on pregnant women and their infants. These data informed the development of clinical tools for healthcare providers caring for and counseling patients with Zika exposure. These tools ensured pregnant women and infants were adequately monitored during the Zika outbreak. Health education

SDS Annual Conference Proceedings 2019. This is an Open Access article distributed under the terms of the Creative Commons AttributionNoncommercial 4.0 Unported License (http://creativecommons.org/licenses/by-nc/3.0/), permitting all non-commercial use, distribution, and reproduction in any medium, provided the original work is properly cited. 
ISDS 2019 Conference Abstracts

materials and clinical tools based on surveillance data should be considered in future emergency responses, particularly when knowledge is rapidly evolving.

\section{References}

Zika Pregnancy Website CDC. https://www.cdc.gov/pregnancy/zika/materials/index.html 